\title{
The Unexpected Capability of Melanin to Split the Water Molecule and the Alzheimer's Disease
}

\author{
Maria del Carmen Arias-Esparza ${ }^{1}$, Ruth Isabel Solís Arias ${ }^{2}$, Paola Eugenia Solís Arias ${ }^{3}$, \\ Martha Patricia Solís Arias ${ }^{4}$, Arturo Solís-Herrera ${ }^{5}$
}

Human Photosynthesis Study Center, Research, Development and Innovation Department, Aguascalientes, Mexico. Email: comagua2000@yahoo.com,comagua2000@gmail.com

Received May $17^{\text {th }}, 2011$; revised July $15^{\text {th }}, 2011$; accepted July 30 2011.

\begin{abstract}
We began a study about the three main causes of blindness in 1990, because their incidence and prevalence have not changed in the last forty years. Twelve years later we concluded that the main source of energy for the human retina is water, not ATP. And this is also true for the entire human body. Water is the main source of energy. The amazing capability of eukaryotic cells to break or dissociate the water molecule was unsuspected to us because it takes $2000^{\circ} \mathrm{C}$ degrees to dissociate water in a laboratory environment, and until today, it was believed that only plants were capable of accomplishing this. Photosynthesis occurs in humans as it does in plants. The water that we drink every day is not just to wash away detritus and toxins; it is not just a cleaner, nor a simple solvent. When our body dissociates the water molecule, cells are able to get their energy from Hydrogen (Hydrogen is the energy carrier that Nature uses the most). Water is our main source of energy. If our body couldn't acquire energy from water, we would need to eat between 50 $170 \mathrm{~kg}(110$ - $374 \mathrm{lbs})$ daily. In any system, when a generalized failure occurs, we must suspect energy first. Parkinson and Alzheimer's Disease are examples of a generalized failure. That explains why it is not uncommon that patients improve dramatically with pharmacological stimulation of the human photosynthesis process. Recall that the brain needs energy not only to grow or to perform its functions, but also to preserve its form and shape. The best energy for human cells is Hydrogen.
\end{abstract}

Keywords: Melanin, Water Dissociation, Energy, Glucose, Alzheimer, Biomass

\section{Introduction}

Many molecular changes have been detected in Alzheimer's disease, but the overarching theme that emerges from the data is that an accumulation of misfolded proteins in the aging brain results in oxidative and inflammatory impairment, like the damage that occurs in other tissues, such as the eye, skin, liver, etc., which in turn leads to energy failure and synaptic dysfunction ${ }^{1}$. However, in light of our new knowledge and discoveries, we can say that glucose is not a source of energy, rather, it is just a source of biomass, therefore, the energy failure happens first, and alterations to the biomass, happen secondly.

Cerebral plaques laden with $\beta$-amyloid peptide $(\mathrm{A} \beta)$ and dystrophic neurites in neocortical terminal fields, as well as prominent neurofibrillary tangles in medial tem

${ }^{1}$ Querfurth, Henry W.M.D., Ph.D., LaFerla, Frank M. Ph.D. Alzheimer's Disease, Mechanisms of Disease. $n$ engl j med 362;4 nejm.org January 28, 2010. poral-lobe structures, are important pathological features of Alzheimer's disease, however, they are not specific. Loss of neurons and white matter, congophilic (amyloid) angiopathy, inflammation, and oxidative damage are also present, but are not pathognomonic of $\mathrm{AD}$. $\mathrm{A} \beta$ peptides are natural products of metabolism consisting of 36 to 43 amino acids. Therefore it seems that the sequence of normal metabolism does occur but with alterations difficult to assign to the relationship activity-structure of the molecules themselves, because their presence is normal at certain levels.

An imbalance between production and clearance, and aggregation of peptides, causes $\mathrm{A} \beta$ to accumulate, and this imbalance more than the final excess of byproducts, may be the initiating factor in Alzheimer's disease. From our point of view, the imbalance is a result of the low levels of energy available. On the other hand, $\mathrm{A} \beta$ spontaneously self-aggregates into multiple coexisting physical forms, a common behavior in many molecules in Nature. 


\section{Amyloid $\beta$}

\subsection{Alzheimer's Disease Is an Energy, and Not a Biomass Troble}

In brain-slice preparations, dimers and trimers of $\mathrm{A} \beta$ are toxic to synapses [1]. The severity of the cognitive defect in Alzheimer's disease correlates with levels of oligomers in the brain, not the total $\mathrm{A} \beta$ burden [2]. Neuronal activation rapidly increases $\mathrm{A} \beta$ secretion at the synapse, a process tied to the normal release of vesicles containing neurotransmitters. Physiologic levels of synaptic $\mathrm{A} \beta$ may dampen excitatory transmission and prevent neuronal hyperactivity [3]. Therefore, molecular changes described in the literature concerning $\mathrm{AD}$ are based in compounds that are not strange to the tissue, the main difference between normal and ill tissue is the amount present, we could say; in general terms, the changes in biomass composition observed in the brain of patients with $\mathrm{AD}$ are not really significant, therefore Alzheimer's Disease is not a biomass problem, it is an energy problem.

Increased oxidative stress, the impaired protein-folding function of the endoplasmic reticulum, and deficient proteasome-mediated and autophagic-mediated clearance of damaged proteins-all of which are also associated with aging-accelerate the accumulation of amyloid and tau proteins in Alzheimer's disease [4]. Therefore, a generalized failure of the cell is evident, and in any system, with a similar behavior, we must think of energy first.

\subsection{Energy and Eukaryotic Cell}

The main source of energy of the eukaryotic cell is water, not ATP. The profound misconception that food, glucose or ATP are the main source of energy has its basis in the lack of knowledge of the hitherto unknown capacity of melanin to split the water molecule [5]. Until today, the fact that human tissues have the capability to take hydrogen from water-the energy carrier by excellence in the whole Universe-arising from the splitting of water, as plants do, was totally unknown before our work.

It was unthinkable that an expensive chemical reaction, from the energetic point of view, such as water dissociation, that requires $2000^{\circ} \mathrm{C}$ in the laboratory to take place, might occur at room temperature in our body. The sole possibility seemed berserk. However, our studies researching the three main causes of blindness allowed us to detect the hitherto unknown fact that melanin is the "human chlorophyll". This amazing compound absorbs photonic energy and transforms it into chemical energy.

\section{The Cycle of Solís-Herrer}

An exhaustively review of melanin is out of the scope of this work, therefore we will write only about the main characteristics of the process. We refer the interested reader to the works of Dr. Paul Meredith and Dr. Dadachova [6].

The reaction in chlorophyll is as follows:

$$
2 \mathrm{H}_{2} \mathrm{O} \longrightarrow 2 \mathrm{H}_{2}+\mathrm{O}_{2}
$$

where as in melanin, the reaction is:

$$
2 \mathrm{H}_{2} \mathrm{O} \longleftrightarrow 2 \mathrm{H}_{2}+\mathrm{O}_{2}
$$

Notice that the reaction in melanin occurs in both directions.

This apparently slight difference between the two is the hallmark of mammal and human life. It is not about dissociating ad infinitum, because hydrogen and the energy that it carries are the basic fuel of the cell. Eukaryotic cells use hydrogen in many ways, starting with the fact that hydrogen is without a doubt the best-known antioxidant. Hydrogen could even reduce oxygen itself and form that strange substance that we call water. Therefore, the main product of the chemical reaction is hydrogen, the energy carrier by excellence in Nature; oxygen is toxic at any level.

Furthermore, melanin could increase oxygen concentration in some tissues, by means of water dissociation, up to $97 \%$ but not more, because at that point the reaction changes direction and begins to reform water, and produces simultaneously with the liquid an orderly flux of electrons that could be registered with appropriate instruments as an undulant direct current.

\subsection{Mitochondria, the Power House of the Eukaryotic Cell}

In many diseases without a defined or specific anatomic or histological substrate, such as Alzheimer's and other neurodegenerative processes of unknown etiology, it's very likely that the ground alteration is a chronic shortage more than an acute shortage of the supply and thereafter availability of hydrogen from the water dissociation process. The main and consistent findings should be mitochondrial alterations, because hydrogen is the precise compound that drives ATP synthase, and when hydrogen supply is not adequate, then the main function of ATP synthase (synthesis of ATP) is impaired. Pioneering biochemical studies have long forged the concept that the mitochondria are the energy powerhouse of the cell. These studies, combined with the unique evolutionary origin of the mitochondria, led the way to decades of research focusing on the organelle as an essential, yet independent, functional component of the cell [7]. However, mitochondria are organelles that function within an integrated reticulum that is continually remodeled by both fusion and fission events; therefore like any other cell component they require energy. So, where is this energy for the mitochondria coming from? 


\subsection{The Answer}

Water dissociation is the answer. We could infer that the way the human photosystem releases energy is completely adequate with many diverse goals. The energy spreads out continuously and symmetrically in all directions through the cytoplasm, night and day. Furthermore, water splitting is not the source of energy for the mitochondria alone, rather every organelle inside any eukaryotic cell needs energy to stay in good function, shape and therefore performance (Figure 1).

In regards to the relationship between water dissociation and ATP formation we have that the hydrogen released by the Human Photosynthesis process it is not solely or exclusively used by the Mitochondria in the Oxidative Phosphorilation process, a set of biochemical reactions not yet well understood. In fact, one of these main reactions, known as Mitchell's Chemo-osmotic Theory, remains a theory decades away from being postulated. Rather, the cell uses hydrogen in many metabolic pathways. To cite an example, hydrogen is, without doubt, the best antioxidant.

The heterogeneity of pathways that could initiate and drive Alzheimer's disease has shown that there is no single linear chain of events, therefore it is a generalized failure, so we must think of a failure in energy first, an impaired water splitting process, and not suspect ATP, blood or glucose metabolic pathway. To complicate matters, some changes are not pathologic but reactionary or protective.

\subsection{The Brain}

Anatomically, the brain is a good example to sustain our finding that the main source of energy is water, not ATP, blood or glucose.

\subsection{Aging-Related Process and Human Photosynthesis}

It remains possible that many of these mechanisms, including the amyloid hypothesis, are minor or wrong, and that some critical aging-related process is the disease trigger (Ref. [1]). Human Photosynthesis could explain this aging-related process, because the capability of the human body begins to decline in the mid-twenties. We lose our capacity to perform the photosynthesis function at its peak during adulthood (which is less than that of embryonic period) at an approximate rate of $10 \%$ with each decade of life after the mid-twenties, and when we reach our fifties it goes into free fall.

The potential risk factor for sporadic Alzheimer's disease, general anesthesia, promotes tau insolubility and $\mathrm{A} \beta$ oligomerization $[9,10]$, and that is congruent with our findings because the water dissociation capacity in the human body is very sensitive to agents with an elevated

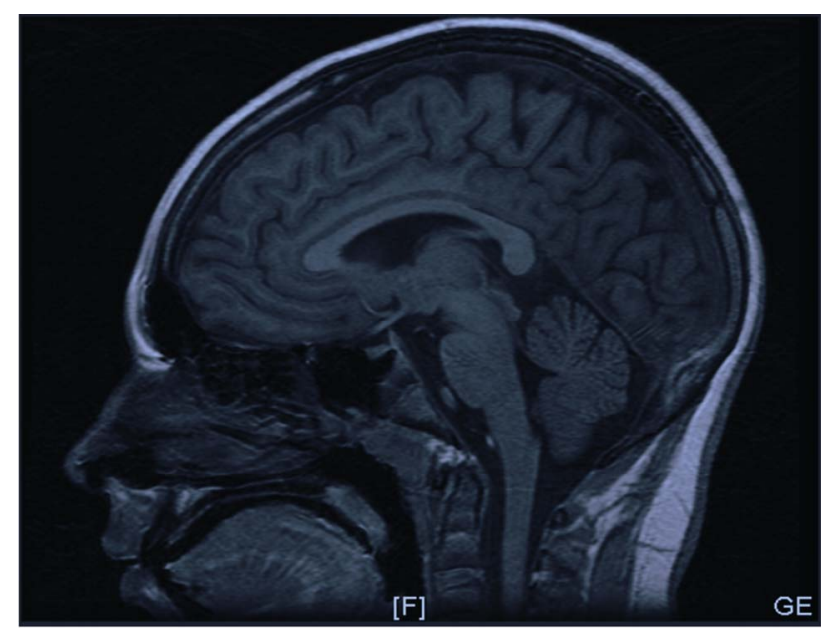

Figure 1. Convolutions of the cerebral cortex have been traditionally explained as a solution to adapt the greatest amount of neuronal tissue in a limited bone-confined intracranial-space. However, from the point of view of our new knowledge, the real goal is to keep every inch, every corner of neuronal tissue in contact with the water of the ventricles and subarachnoid space. The main source of energy is the water of the CSF in the ventricles and subarachnoid space, not the blood vessels [8].

apparent distribution volume, such as anesthesia agents and antidepressants.

The axonal-transport deficits are an internal derangement that is probably an effect rather than a cause of Alzheimer's Disease; therefore it is not hard to believe that it could be normalized with an adequate level of human photosynthesis, or water splitting process, because the first requirement of any process in our body is energy, undoubtedly.

The fact that Glucose intolerance and type 2 diabetes are considered to be risk factors for dementia [11] is consistent with our new knowledge that glucose is only the main source of biomass, but the usual order of events are alterations in energy first, and then biomass disorders in second place.

Iron poisons melanin itself, so elevated levels of this divalent transition metal are linked with neuro-degeneration in several ways [12].

The higher serum glucose levels that are common in normal aging directly damage hippocampal structures [13], probably because greater amount of water than normal is required by the eukaryotic cell to move the glucose molecule, lessening the availability of water to produce energy by means of dissociation of the water molecule.

Something similar happens in the imbalanced expression of low-density lipoprotein receptor-related proteins and receptors for advanced glycation end products, proposed by the neurovascular uncoupling hypothesis [14]. 


\section{Oxidative Stress}

Dysfunctional mitochondria release oxidizing free radicals, and in the brain of Alzheimer's disease patients and in the normal aging brain, they cause considerable oxidative stress [15]. However, mitochondria cannot be dysfunctional if energy levels are adequate, because after billions of years of evolution these organelles know very well what they have to do. Experimental models show that markers of oxidative damage precede pathological changes [16]. $\mathrm{A} \beta$, a potent generator of reactive oxygen species [17] and reactive nitrogen species [18], is a prime initiator of this damage, but the best antioxidant, without question, is the diatomic hydrogen, the main product of the water dissociation process performed by the Human Photosystem, composed by Light/Melanin/Water, arranged in order of abundance in Nature.

We can demonstrate that energy from water is a very important source of hydrogen. The amount of food that we ingest every day (about 700 g) cannot satisfy our daily requirements of ATP (11000 calories a minute). And devices such as this photo-electrochemical self-renewable cell, which can light up LEDs during years, day and night, have demonstrated the flux of electrons. (Figure 2)

\section{Therapeutic Results}

In our clinic we have treated with extraordinary results several patients with Alzheimer's Disease (Figure 3 and 4), as well as patients with many other diseases by enhancing the Human Photosynthesis process. Because Human Photosynthesis is the chemical reaction that fully explains the origin of life, all biochemical processes, tissues, organs and systems that comprise the human body have been created after it and are owing to the energy transduced by the Human Photosystem. The basis of the treatment is the enhancement of the water splitting process with pharmacological agents developed by our team. As we increase the available energy in the cells, and because it is released symmetrically in all directions, all intracellular organelles, even the nucleus, as well as biochemical compounds present in the cytoplasm and nucleosol, that require energy to be transformed, activated, or modified benefit in many ways in order to perform their function adequately.

\section{Conclusions}

The discovery that glucose is a source of biomass, and not a source of energy, is groundbreaking knowledge. Therefore Alzheimer's Disease is an energy problem, not a biomass problem. Any biochemical reaction requires energy as a first step. Thereafter all changes in atoms, molecules, cells or tissues also need energy to continue with all the different subset of reactions, therefore, en

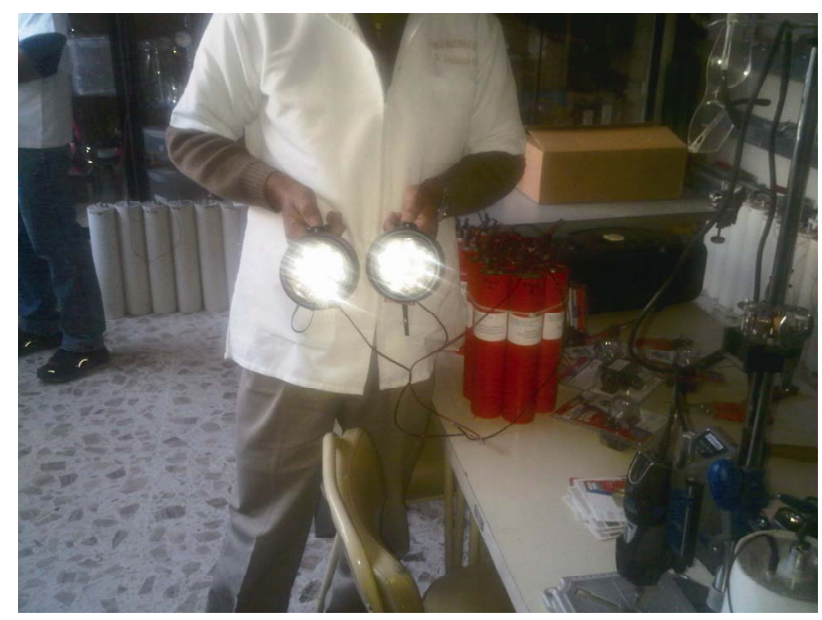

Figure 2. The image shows an LED arrangement energized with a device that we have developed to demonstrate the biological event in the test tube [19].

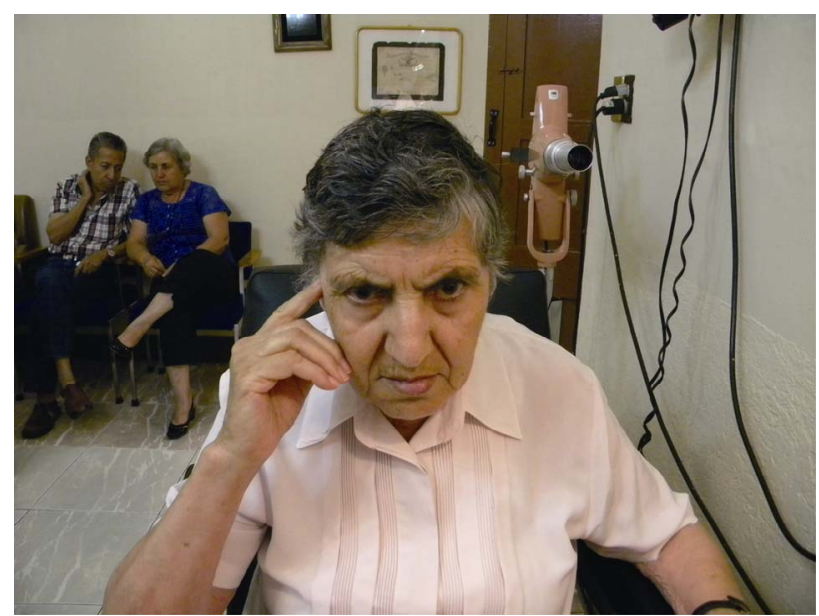

Figure 3. Shows an Alzheimer's Disease patient on the first examination, notice the aggressive look on her face.

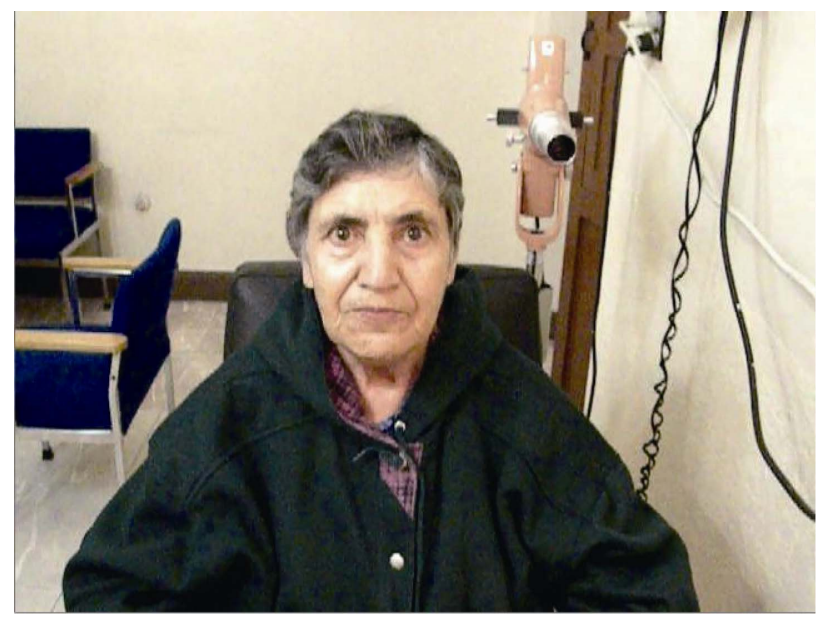

Figure 4. Same patient five weeks after starting treatment using Human Photosynthesis enhancer. 
ergy is constantly needed. In other words, energy is required at the begining, during and after the reaction, i.e. at all times.

In $\mathrm{AD}$ the chronic low levels of water dissociation mean a chronic shortage of energy that will be manifested several ways along the path of evolution of the disease. Histological findings are in accordance with a generalized more than a punctual failure, and in any system with this type of alteration we must think of energy first.

\section{REFERENCES}

[1] I. Klyubin, V. Betts, A. T. Welzel, et al., “Amyloid Beta Protein Dimer-Containing Human CSF Disrupts Synaptic Plasticity: Prevention by Systemic Passive Immunization,” The Journal of Neuroscience, 2008, Vol. 28, No. 16, pp. 4231-4237. doi:/10.1523/JNEUROSCI.5161-07.2008

[2] L. F. Lue, Y. M. Kuo, A. E. Roher, et al., "Soluble Amyloid Beta Peptide Concentration as a Predictor of Synaptic Change in Alzheimer's Disease," The American Journal of Pathology, Vol. 155, No. 3, 1999, pp. 853-62. doi:/10.1016/S0002-9440(10)65184-X

[3] F. Kamenetz, T. Tomita, H. Hsieh, et al., "APP Processing and Synaptic Function,” Neuron, Vol. 37, No. 6, 2003, pp. 925-937. doi:/10.1016/S0896-6273(03)00124-7

[4] J. Carter and C. F. Lippa, "Beta-Amyloid, Neuronal Death and Alzheimer's Disease," Current Molecular Medicine, Vol. 1, No. 6, December 2001, pp. 733-737. doi:/10.2174/1566524013363177

[5] A. Solís-Herrera, M. de C. Arias-Esparza, R. I. SolísArias, P. E. Solís-Arias and M. P. Solís-Arias, "The Unexpected Capacity of Melanin to Dissociate the Water Molecule Fills the Gap Between the Life Before and After ATP,” Biomedical Research, Vol. 21, No. 2, 2010, pp. 224-226.

[6] E. Dadachova, R. A. Bryan and O. C. Howell, "The Radioprotective Properties of Fungal Melanin Are a Function of Its Chemical Composition, Stable Radical Presence and Spatial Arrangement,” Pigment Cell \& Melanoma Research, Vol. 21, No. 2, 2008, pp. 192-199.

[7] M. P. SolísArias, “The Unexpected Capacity of Melanin to Dissociaolecule Fills the Gap between the Life before and after ATP,” Biomedical Research, Vol. 21, No. 2, 2010, pp. 224-226.

[8] A. Solís-Herrera, Ma del C. Arias-Esparza, et al., "The Pharmacologic Intensification of the Water Dissociation (Human Photosynthesis) and Its Effect over Tissues Affected by Bloodshed of Diverse Etiology," International Journal of Clinical Medicine, Vol. 2, No. 2, 2011, pp. 332-338. doi:/10.4236/ijcm.2011.23058
[9] E. Planel, A. Bretteville, L. Liu, et al., “Acceleration and Persistence of Neurofibrillary Pathology in a Mouse Model of Tauopathy Following Anesthesia,” The FASEB Journal, Vol. 23, No. 8, 2009, pp. 2595-2604. doi:/10.1096/fj.08-122424

[10] Z. Xie, D. J. Culley, Y. Dong, et al., “The Common Inhalation Anesthetic Isoflurane Induces Caspase Activation and Increases Amyloid Beta-Protein Level in Vivo," Annals of Neurology, Vol. 64, No. 6, 2008, pp. 618-267. doi:/10.1002/ana.21548

[11] Z. Arvanitakis, R. S. Wilson, J. L. Bienias, D. A. Evans and D. A. Bennett, "Diabetes Mellitus and Risk of Alzheimer Disease and Decline in Cognitive Function," $\mathrm{Ar}$ chives of Neurology, Vol. 61, No. 5, 2004, pp. 661-666. doi:/10.1001/archneur.61.5.661

[12] M. A. Lovell, J. D. Robertson, W. J. Teesdale, J. L. Campbell, W. R. Markesbery, "Copper, Iron and Zinc in Alzheimer's Disease Senile Plaques,” Journal of the Neurological Sciences, Vol. 158, No. 1, 1998, pp. 47-52. doi:/10.1016/S0022-510X(98)00092-6

[13] W. Wu, A. M. Brickman, J. Luchsinger, et al., "The Brain in the Age of Old: The Hippocampal Formation Is Targeted Differentially by Diseases of Late Life,” Annals of Neurology, Vol. 64, No. 6, 2008, pp. 698-706. doi:/10.1002/ana.21557

[14] R. Deane and B. V. Zlokovic, "Role of the Blood-Brain Barrier in the Pathogenesis of Alzheimer's Disease," Current Alzheimer Research, Vol. 4, No. 2, 2007, pp. 191-197. doi:/10.2174/156720507780362245

[15] P. F. Good, P. Werner, A. Hsu, C. W. Olanow and D. P. Perl, "Evidence of Neuronal Oxidative Damage in Alzheimer's Disease,” The American Journal of Pathology, Vol. 149, No. 1, 1996, pp. 21-28.

[16] A. Nunomura, G. Perry, G. Aliev, et al., "Oxidative Damage Is the Earliest Event in Alzheimer Disease," Journal of Neuropathology \& Experimental Neurology, Vol. 60, No. 7, 2001, pp. 59-67.

[17] K. Hensley, J. M. Carney, M. P. Mattson, et al., “A Model for Beta-Amyloid Aggregation and Neurotoxicity Based on Free Radical Generation by the Peptide: Relevance to Alzheimer Disease," Proceedings of the $\mathrm{Na}$ tional Academy of Sciences of the USA, Vol. 91, No. 8, 1994, pp. 3270-3274. doi:/10.1073/pnas.91.8.3270

[18] C. K. Combs, J. C. Karlo, S. C. Kao and G. E. Landreth, "Beta-Amy-Loid Stimulation of Microglia And Monocytes Results in TNFalpha-Dependent Expression of Inducible Nitric Oxide Synthase and Neuronal Apoptosis," The Journal of Neuroscience, Vol. 21, No. 4, 2001, pp. 1179-1188.

[19] A. Solis-Herrera, M. E.Lara and L. E. Rendón, “The Photoelectrochemical Properties of Melanin,” Nature Precedings, 2007. hdl:10101/npre.2007.1312.1 